\title{
Increased sputum amino acid concentrations and auxotrophy of Pseudomonas aeruginosa in severe cystic fibrosis lung disease
}

\author{
Stephen R Thomas, Anjana Ray, Margaret E Hodson, Tyrone L Pitt
}

\begin{abstract}
Background-Pseudomonas aeruginosa may undergo a phenotypic change from the wild (prototrophic) type to an auxotrophic phenotype in the course of respiratory infection in patients with cystic fibrosis. The clinical significance of this is unclear. A study was undertaken to investigate whether the presence of auxotrophs of $P$ aeruginosa in the sputum of patients with cystic fibrosis correlated with severity of respiratory disease, and whether increased sputum concentrations of amino acids were associated with the emergence of these forms.

Methods-Sixty adult patients with cystic fibrosis, colonised by $P$ aeruginosa, were recruited and baseline clinical data including lung function were recorded. Serial sputum samples were obtained before, during, and after infective exacerbations where possible. These samples were used for routine microbiological culture, assessment of auxotrophy of $P$ aeruginosa, measurement of amino acid content, and neutrophil elastase assay.
\end{abstract}

Results-Auxotrophy was common in patients with cystic fibrosis and $20(33 \%)$ had a mean percentage auxotroph count of more than $50 \%$ total $\mathrm{cfu} / \mathrm{ml}$. The mean percentage auxotroph count was inversely correlated with forced expiratory volume in one second $\left(\mathrm{FEV}_{1} ; \tau=-\mathbf{0 . 1 9 4 ,} \mathbf{p}=\mathbf{0 . 0 3 1}\right)$. The median sputum amino acid concentration of the group was $12.5 \mathrm{mmol} / 1$ (range 0.13-40.6). The mean amino acid concentration in 33 subjects during infective exacerbations was $18.2 \mathrm{mmol} / 1$ (95\% CI 15.1 to 21.3) compared with $12.3 \mathrm{mmol} / 1$ (95\% CI 9.8 to 14.8$)$ when well $(p=0.001)$. The amino acid content of sputum was inversely correlated with $\mathrm{FEV}_{1}(\tau=-0.253, \mathrm{p}=0.005)$

Conclusions-P aeruginosa frequently exhibits auxotrophy in patients with cystic fibrosis, particularly in those with severe underlying pulmonary disease. The sputum amino acid content of patients with cystic fibrosis is high during infective exacerbations and correlates with pulmonary disease severity.

(Thorax 2000;55:795-797)

Keywords: cystic fibrosis; Pseudomonas aeruginosa; auxotrophy

Most older patients with cystic fibrosis are colonised by Pseudomonas aeruginosa and ac- quisition of this pathogen is associated with an accelerated decline in respiratory function. ${ }^{1}$ Recently, auxotrophic phenotypes of $P$ aeruginosa have been described. ${ }^{2}$ Auxotrophs are dependent upon specific amino acids for growth, ${ }^{3}$ whereas the wild type prototroph is able to grow on a minimal medium containing mineral salts and glucose as the sole carbon source. An earlier small study of adult cystic fibrosis patients found an increase in the proportion of auxotrophs to prototrophs during infective exacerbations and in those with more severe disease. ${ }^{4}$ Genotype analysis of pairs of isolates from individual patients suggests that the auxotrophs originate from pre-existing prototrophs ${ }^{3}$ and sputum extracts from patients with cystic fibrosis are able to promote their growth. ${ }^{5}$

The purpose of this study was to investigate the clinical significance of the auxotrophic phenotype of $P$ aeruginosa in patients with cystic fibrosis. Specifically, we tested the hypothesis that the auxotrophic phenotype of $P$ aeruginosa is found in patients with more severe disease (especially during infective exacerbations) and that this phenomenon may be driven by increased concentrations of amino acids in the bronchial secretions of these patients.

\section{Methods}

Adult patients attending the Cystic Fibrosis Department at the Royal Brompton Hospital were recruited into the study. All patients were colonised with $P$ aeruginosa but those also colonised with Burkholderia cepacia were excluded. The study was approved by the Royal Brompton Hospital ethics committee and informed consent was obtained from each subject.

At the time of recruitment the forced expiratory volume in one second $\left(\mathrm{FEV}_{1}\right)$ and forced vital capacity (FVC) were recorded and the percentage predicted values were calculated using established reference values. ${ }^{67}$ The frequency of recorded infective exacerbations in the preceding year was noted. An infective exacerbation was defined as an episode for which the patient was treated with oral or intravenous antibiotics for any four of the following 10 signs or symptoms: change in sputum volume; new or increased haemoptysis; increased cough; increased dyspnoea; malaise, fatigue or lethargy; temperature above $38^{\circ} \mathrm{C}$; anorexia or weight loss; change in physical examination of chest; $10 \%$ decrease in $\mathrm{FEV}_{1}$ from a previously recorded value; and radiographic changes indicative of pulmonary infection. After recruitment patients were followed 
Table 1 Clinical characteristics of patients with cystic fibrosis

Number of subjects

Male (\%)

Median (range) age (years)

Median (range) $\mathrm{FEV}_{1}$ (\% predicted)

Median (range) no. exacerbations in previous year

Median (range) sputum samples per patient

Median (range) duration of study (months)

over subsequent months in order to obtain further sputum samples during infective exacerbations and when well.

A sputum sample was obtained when patients were recruited and subsequently at the time of outpatient attendance or admission to hospital. Manually homogenised sputum was serially diluted $\left(10^{-2}\right.$ to $\left.10^{-8}\right)$ in Ringer's solution and $100 \mu \mathrm{l}$ aliquots of each dilution were spread uniformly on both a minimal salt medium with $0.2 \%$ glucose which supports only the growth of prototrophs, and Kings "A" agar which supports both nutritional types. After aerobic culture at $37^{\circ} \mathrm{C}$ for 48 hours the total colony count on each plate and the proportions of auxotrophs and prototrophs were determined. Isolates that produced the characteristic pigment pyocyanin were accepted to be $P$ aeruginosa and non-pigmented isolates were tested for their ability to oxidise glucose, produce cytochrome oxidase, hydrolyse arginine, and reduce nitrate.

An aliquot of the sputum sample used for microbiology was stored at $-70^{\circ} \mathrm{C}$ and subsequently assayed for total amino acid content by HPLC using the Waters AccQTag amino acid analysis method (Waters Ltd, Watford, Hertfordshire, UK).

Forty two sputum samples from 33 patients were available for the measurement of neutrophil elastase. An equal volume of phosphate buffered saline $\mathrm{pH} 7.2$ was added to the sample which was gently mixed and, after centrifugation at $50000 \mathrm{~g}$, the supernatant was separated and stored at $-70^{\circ} \mathrm{C}$. An assay in which 7-amino-4-methylcoumarin is detected fluoro-

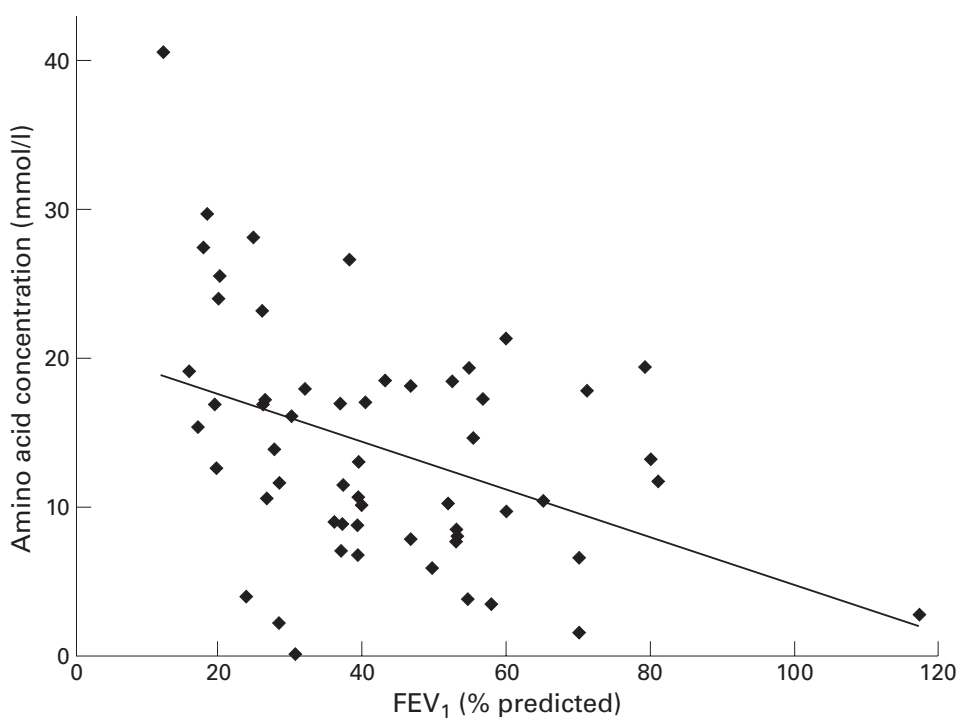

Figure 1 Correlation between amino acid concentration in sputum (mmol/l) and FEV \% predicted in 57 patients with cystic fibrosis. Where more than one sputum sample was available for amino acid analysis, the mean value for individual patients was calculated ( $p$ $=0.004$, Kendall's $\tau$ ). metrically after cleavage from MeOSuc-AlaAla-Pro-Val-7-amino-4-methylcoumarin (Genosys, Cambridgeshire, UK) by neutrophil elastase was used.

The Statistical Package for Social Sciences (SSPS for Windows version 8.0.0) was used for data analysis. Non-parametric analyses were used as indicated. All reported $\mathrm{p}$ values were considered significant at the 0.05 level.

\section{Results}

The characteristics of the patients are shown in table 1. A total of 60 eligible patients were recruited. There was a wide range of disease severity within the group, as illustrated by lung function and documented infective exacerbations in the preceding year. A total of 231 sputum samples yielded $P$ aeruginosa on culture.

Considerable variability in the proportion of the auxotrophic phenotype of $P$ aeruginosa was observed, both between individuals and in different samples from the same individuals. Auxotrophy was, however, common; 20 subjects $(33 \%)$ had a mean percentage auxotroph count of more than $50 \%$ of the total colony count and $19(32 \%)$ had counts of $10-50 \%$.

The association between infective exacerbations and auxotrophy was investigated in 39 subjects for whom sputum samples were available during infective exacerbations and when well. The median (range) percentage of auxotrophs during infective exacerbations was $16.5 \%(0-100 \%)$ compared with $15.3 \%$ (0$100 \%$ ) when well ( $\mathrm{p}=0.41$, Wilcoxon). A significant correlation was observed between disease severity and auxotroph counts $(\tau=$ $-0.194, p=0.031$, Kendall's $\tau$ ). Auxotrophy was therefore more common in those with severe lung disease than in those with mild or moderate disease.

The median sputum amino acid concentration was $12.5 \mathrm{mmol} / 1$ (range 0.13-40.6). There were 33 individuals for whom paired samples for amino acid assay were available during infective exacerbations and when well. The mean amino acid concentration during infective exacerbations was $18.2 \mathrm{mmol} / 1$ (95\% CI 15.1 to 21.3 ) compared with $12.3 \mathrm{mmol} / \mathrm{l}$ (95\% CI 9.8 to 14.8$)$ when well $(\mathrm{p}=0.001$, Wilcoxon). The correlation between amino acid content and disease severity as assessed by $\mathrm{FEV}_{1}$ was -0.26 (Kendall's $\tau, \mathrm{p}=0.004$ ) and indicated that amino acid concentrations in sputum were higher in those with more severe disease (fig 1). The correlation between sputum neutrophil elastase and amino acid concentration for 42 samples from 33 patients was $0.525(p=0.002)$. No significant correlation was observed between the amino acid content of sputum and auxotroph count $(\mathrm{p}=$ $0.78)$.

\section{Discussion}

This study shows that auxotrophy of $P$ aeruginosa is commonly observed in the sputum of patients with cystic fibrosis. The mechanism of selection and overgrowth of auxotrophs in the lung is not clear, and this study did not show a direct correlation between sputum amino acid concentration and auxotrophy. A common 
energy saving strategy employed by bacteria is the downregulation of a metabolic pathway that results in the generation of a substance that occurs freely in the environment. Amino acid synthesis is a metabolically expensive process and therefore bacteria that lose the ability to synthesise an amino acid that is locally abundant may have a selective advantage over prototrophs and outgrow them in mixed culture. The lack of correlation between sputum amino acid concentrations and auxotrophy is possibly explained by the fact that amino acid concentrations in most cystic fibrosis airways are above that required by most auxotrophic stains.

There was no increase in the proportion of auxotrophs during infective exacerbations. This was unexpected because a previous study had suggested that this did occur, ${ }^{4}$ and also because the proteolytic activity measured in sputum from cystic fibrosis patients increases during infective exacerbations. We did, however, observe considerable fluctuations in the proportion of auxotrophs without a perceptible change in the clinical state of the patients, and it may be that changes during infective exacerbations were not detectable in view of this substantial baseline variability. As the proportion of auxotrophs of $P$ aeruginosa does not significantly increase during infective exacerbations, it seems likely that this phenotype does not play a causative role in the onset of infective exacerbations.

Raised concentrations of amino acids have previously been observed in patients with cystic fibrosis. ${ }^{5}$ This study adds to this by demonstrating an association between amino acid concentration and severity of pulmonary disease. There are a number of possible explanations for raised amino acid concentrations in the lungs of cystic fibrosis patients. It has been suggested that the concentration of amino acids in alveolar and bronchial surface fluids depends upon active epithelial transport, plasma exudation, and protease activity in lung tissue and surface liquid. There is some evidence that, in conditions associated with acute inflammation, plasma amino acid concentrations are reduced $^{8}$; it therefore seems unlikely that increased levels of amino acids simply reflect increased plasma concentrations. Proteolytic enzymes released from neutrophils and the activity of bacterial exoproteases may result in the breakdown of proteins to small peptides with subsequent cleavage to amino acids by inner membrane associated peptidases. Although such a mechanism could account for the correlation between neutrophil elastase and amino acid concentration in sputum, in vitro experiments have not identified free amino acids in oligopeptide containing medium in which $P$ aeruginosa was growing. ${ }^{9}$

The lack of correlation between auxotrophy and sputum amino acid concentration could be explained by the confounding effect of oligopeptides in sputum which were not assayed in this study. Membrane associated peptidases have been shown to allow methionine dependent auxotrophs of $P$ aeruginosa to grow when methionine is available only within di-, tri-, tetra- and pentapeptides. ${ }^{9}$

This work was funded in part by the Cystic Fibrosis Research Trust. The authors are grateful to Jackie Turner for statistical advice and Professor D M Geddes for his critical review of the manuscript.

1 Pamukcu A, Bush A, Buchdahl R. Effects of Pseudomonas aeruginosa colonization on lung function and anthropometric variables in children with cystic fibrosis. Pediatr Pulmonol 1995;19:10-15.

2 Taylor R F, Hodson ME, Pitt TL. Auxotrophy of Pseudomonas aeruginosa in cystic fibrosis. FEMS Microbiol Pseudomonas aerugin 1992;71:243-6.

3 Barth AL, Pitt TL. Auxotrophic variants of Pseudomonas aeruginosa are selected from prototrophic wild-type strains in respiratory infections in patients with cystic fibrosis. $\mathcal{F}$ Clin Microbiol 1995;33:37-40.

4 Taylor RF, Hodson ME, Pitt TL. Adult cystic fibrosis: association of acute pulmonary exacerbations and increasing severity of lung disease with auxotrophic mutants of Pseudomonas aeruginosa. Thorax 1993;48:1002-5.

5 Barth AL, Pitt TL. The high amino-acid content of sputum from cystic fibrosis patients promotes growth of auxotrophic Pseudomonas aeruginosa. F Med Microbiol 1996;45: 110-9.

6 Rosenthal M, Bain SH, Cramer D, et al. Lung function in white children aged 4 to 19 years: I. Spirometry. Thorax 1993;48:794-802.

7 Quanjer PH, Tammeling GJ, Cotes JE, et al. Lung volumes and forced ventilatory flows. Report of Working Party on Standardization of Lung Function Tests, European Community for Steel and Coal. Official Statement of the European Respiratory Society. Eur Respir 7 1993;16(Suppl):540 .

8 Pouw EM, Schols AM, Deutz NE, et al. Plasma and muscle amino acid levels in relation to resting energy expenditure and inflammation in stable chronic obstructive pulmonary disease. Am f Respir Crit Care Med 1998;158:797-801.

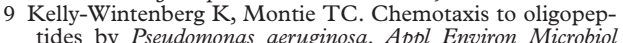
tides by Pseudomonas aeruginosa. Appl Environ Microbiol 